\title{
Use of IVIVC to Optimize Generic Development
}

\author{
J.-M. Cardot*, G. Garrait, and E. Beyssac \\ Auvergne University, UFR Pharmacie, EA4678, Biopharmaceutical Department, 28 \\ Place H. Dunant, BP 38, 63001 Clermont-Ferrand, France
}

e-mail:j-michel.cardot@udamail.fr

\section{ABSTRACT}

During the development of a generic sustained-release dosage form, an in vivo pilot study is often performed. This pilot study could be the starting point to set up an IVIVC. Thanks to the relationship established between in vitro data and in vivo curve, a predictive in vitro model could be determined allowing calculation of an optimal dissolution curve. Based on those available tools, using a design of experiment, the formulation can be optimized with a minimum of effort. The target is to develop a formulation whose dissolution is as close as possible to the calculated optimal dissolution curve. This approach demonstrates that it is possible to improve the selection of the best formulation and minimize the risk in the bioequivalence final study using the in vivo profile observed during the pilot study and the IVIVC.

KEYWORDS: In vitro-in vivo correlation (IVIVC); dissolution curve; prediction; formulation optimization.

\section{INTRODUCTION}

$\mathrm{n}$ vitro-in vivo correlations (IVIVC) can be summarized as relationships observed between parameters or curves derived from in vitro (dissolution data on the $X$-axis: full dissolution curve or dissolution parameters) and in vivo (absorption curve or bioavailability or bioequivalence parameters on the $Y$-axis). The underlying assumption with correlations is that the rate-limiting factor in vivo must be reproducible in vitro and must not be linked with physiological factors (i.e., permeability/permeation through the membrane) but rather with the different rates of drug release from the drug dosage form (DDF) or to a lesser extent, with the solubilization of the active pharmaceutical ingredient (API). All of these correlations imply linear non-saturable pharmacokinetic processes (1-3). For the highest level of IVIV correlation (Level A), the absorption curves presented as the percentage of the fraction of dose absorbed versus time are put in relation to the dissolution curve obtained in vitro. This absorption curve represents the slowest of all the phenomena that take place prior to and during absorption, such as drug release from the pharmaceutical dosage form, dissolution in the gastrointestinal fluid, and permeability through intestinal membranes. For a sustained-release dosage form, it mainly represents the release from the dosage form.

IVIVC stresses the importance of the in vitro dissolution (4-14), which must directly reflect the release and dissolution of the drug from the dosage form and all the aspects related to the formulation work. In contrast to in vivo studies, in vitro methods can be adapted to the dosage form developed as different apparatus could be used (USP Apparatus 1-4) with various media $(\mathrm{HCl}$, buffer solutions, added surfactants or enzymes, complexation media: FaSSIF and FeSSIF), using pre-established technical parameters (e.g., volume, rate, flow). Any method (combination of apparatus, dissolution medium, and flow rate or rotation

${ }^{*}$ Corresponding author. speed) able to discriminate between formulations can be used. To compare the results, the same method must be used to compare all the formulations developed.

In case of sustained-release formulations, formulation work plays a key role in the development of the dosage form, and IVIVCs are of great interest. All the IVIVC work performed is only valid in the context of the studied formulations; in other words, all the modified and optimized dosage forms must be manufactured within the same company, with similar processes, and have the same release mechanism (even with different rates) and route of administration $(5-11,15)$. For instance, it would not be acceptable to use the IVIVC generated on the originator formulation in the submission of a generic dossier.

The aim of this paper is to show how IVIVC can be established using results of a pilot study and how optimization of a generic formulation can be done based on the in vivo results of the originator.

\section{PILOT STUDY: IDEAL STARTING POINT TO ESTABLISH IVIVC}

Often during the development of slow-release formulations of generics, different technology from that of the originator could be used for the generic for either patent or price issues. In this case, the in vivo behavior of the prototype is tested using a pilot study to estimate the best option. Once the best option or technology is selected, the formulation is optimized using the same technology. For a sustained-release formulation, release from the pharmaceutical dosage form is the limiting factor, and if no other physiological limiting absorption processes takes place, IVIVC could be investigated based on the pilot results to optimize efficiently the formulation through a design of experiment (DOE) based on the target in vitro dissolution profile. The aim of the IVIVC in this case is to adequately estimate the target dissolution curve of the optimized formulation based on the expected in vivo 
results that would mimic the reference formulation and then predict the in vivo risk.

From the in vivo pilot study data and all in vitro results, a classical IVIVC approach could be used $(1-3,16,17)$, and this process is performed in two main steps: (1) calculation of the in vivo in put function (absorption) by a deconvolution technique and (2) link with in vitro dissolution data using a regression method. An alternative method would be to use a direct convolution technique (18-21) that will allow the investigator to treat all the information in a single process using specific algorithms and software derived from population pharmacokinetics. The first approach using a two-step method will be described.

\section{EXAMPLE OF USE OF LEVEL A IVIVC TO OPTIMIZE A GENERIC FORMULATION}

The most common process for developing a generic is to develop formulations with different release rates, such as slow, medium (target), and fast release, based on a dissolution test that is expected to be predictive (5-11). The next in vivo step is a pilot study to test the prototypes and to obtain plasma concentration profiles for these formulations. If none of the formulations is the optimal one, or if the pilot study failed to confirm that one of the prototypes is bioequivalent, the data can still be used to develop an IVIVC. From the in vitro and in vivo profiles, the dissolution and absorption time courses are obtained using an appropriate technique for each formulation. These results lead to the establishment of the IVIVC model.

As an example, the formulations ( $\mathrm{A}, \mathrm{B}$, and $\mathrm{C})$ tested in a pilot study gave in vitro results in accordance with the requirements (fast, medium, and slow release) as presented in Figure 1. Based on those good results, an in vivo pilot study was performed on the reference and the three different formulations developed (Figure 2). Unfortunately, compared with the reference product, the in vivo results did not reflect the forecasted in vitro results. Despite the fact that the in vivo profiles were in accordance with the expectations (fast, medium, and slow profiles), the target formulation was far from the reference one. The in vitro dissolution method was not predictive. The next step of the development was to analyze the non-optimal results of the in vivo pilot to understand the problem and to develop a better in vitro dissolution method. First, based on the in vivo data, the in vivo absorption of each formulation curve was calculated using a deconvolution method (Figure 3). The analysis of the absorption curves clearly shows that the in vivo behavior of the reference was different from that of the test formulations. For the calculation of the absorption curve, the formulation C (slow) produced questions. The terminal part of the curve is different from those of all the other formulations, and a "flip-flop" model could be discussed (22). In a flip-flop model, rate of absorption approximates rate of elimination; in a simplified sense, rate of absorption is the rate-limiting step in the sequentialparallel processes of drug absorption, distribution, and elimination. This could be classically obtained for a slow sustained-release formulation. The possibility of a flip-flop model for formulation $C$ was considered, and the absorption profile was determined based on such assumption (Figure 3 flip-flop profile). As for a slow-release formulation, the absorption curve reflects the slowest of the phenomena: release, dissolution of the API, and permeation. It could be thought that the differences between the developed and the reference formulations are due to an inadequate in vivo release of the test formulations. In addition, there is a great difference between the in vitro dissolution kinetics performed and the in vivo absorption curves calculated from in vivo profiles, at least in terms of rate of dissolution/ absorption (Figure 1 vs 3).

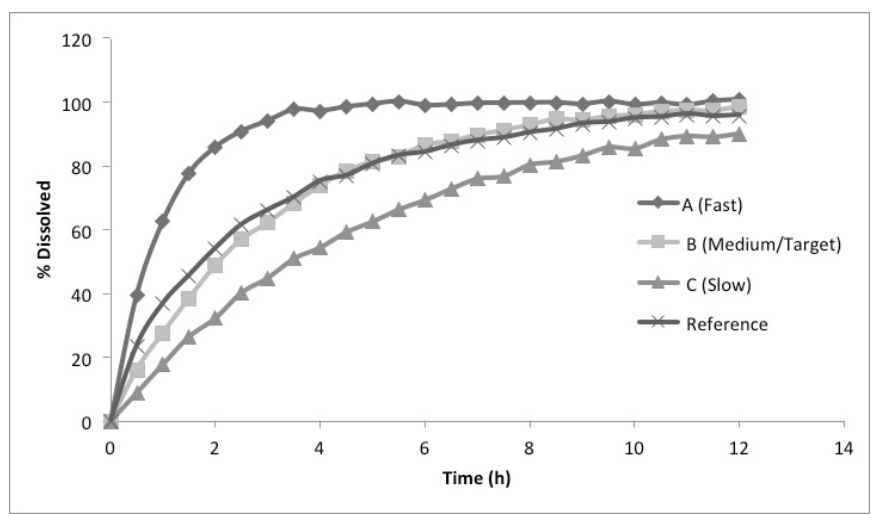

Figure 1. In vitro dissolution percentage dissolved of test formulations A (Fast), B (Medium/Target), and C (Slow) compared with the reference formulation.

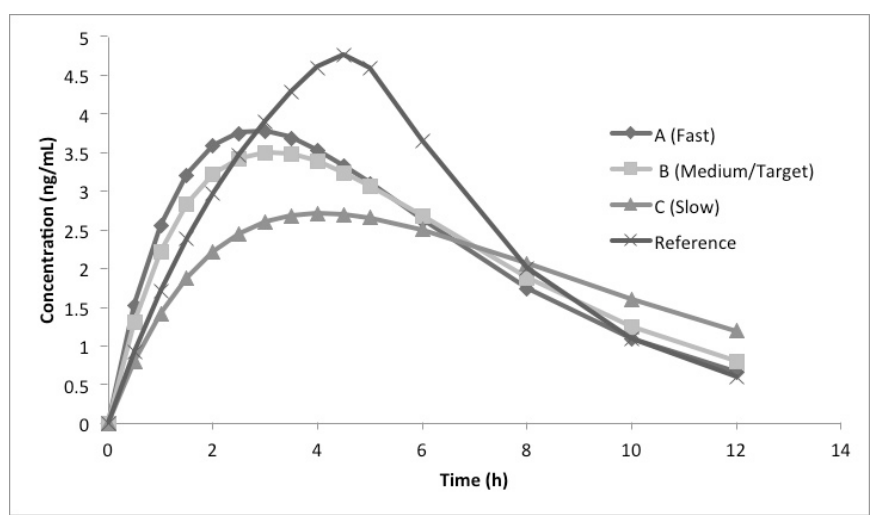

Figure 2. In vivo plasma concentration-time curves of test formulations A (Fast), B (Medium/Target), and C (Slow) compared with the reference formulation.

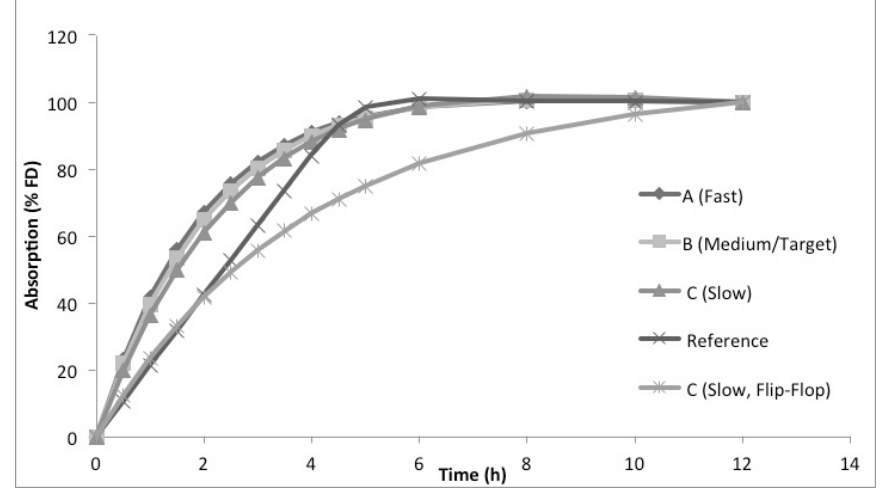

Figure 3. In vivo absorption curves of test formulations A (Fast), B (Medium/Target), and C (Slow and Flip-flop) compared with the reference formulation. 
Based on the in vitro dissolution and in vivo absorption data available for test formulations, a linear relationship was investigated (Figure 4). The in vitro data are presented on the $X$-axis (as it usually exhibited less variability), and the in vivo on the $Y$-axis. In an ideal situation, the in vitro data should reflect in vivo behavior (one-to-one relationship between the in vivo and the in vitro data), leading to a linear relationship with a slope close to 1 and an intercept not different from 0 indicating a direct proportionality. In all other cases, the nature of the relationship between the in vivo and the in vitro data needs further investigation.

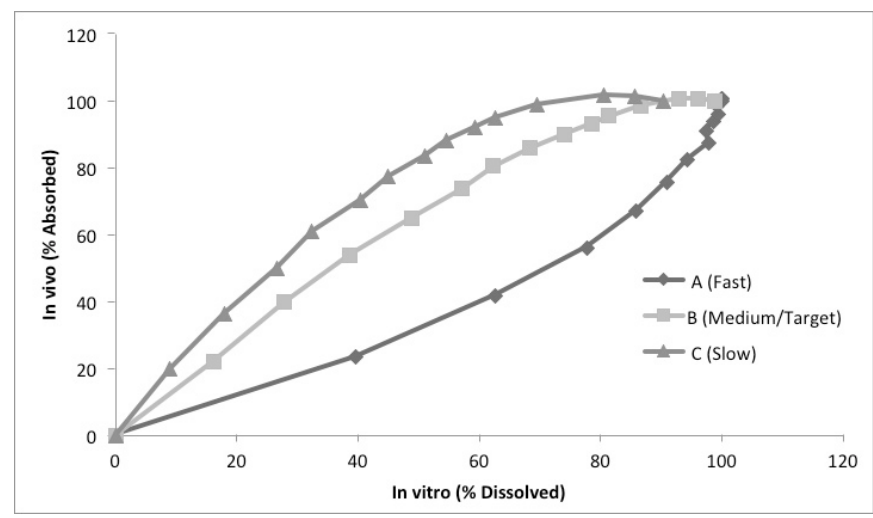

Figure 4. First attempt to establish an IVIVC between $\%$ dose absorbed from in vivo profiles vs. in vitro \% dose dissolved for test formulations A (Fast), B (Medium/Target), and C (Slow).

Looking at the percentage absorbed (\%FD) versus percentage of drug dissolved over time (Figure 4), as a first attempt to build an IVIVC, a relation close to a linear one for $\mathrm{B}$ and $\mathrm{C}$ and a nonlinear relationship for $\mathrm{A}$ are highlighted. Those differences indicate that either the kinetic order of the input in vivo and in vitro are different (for example the dissolution may appear to be zero order and the in vivo input first order) or the rates of absorption/dissolution are different between in vivo and in vitro. In those cases, a direct IVIVC is not possible, and a new dissolution technique that matches the vivo data must be developed. Changing the in vitro conditions (apparatus, dissolution medium, etc.) should allow development of a predictive dissolution method. The results of the new dissolution data for all three test and the reference formulations are presented in Figure 5.

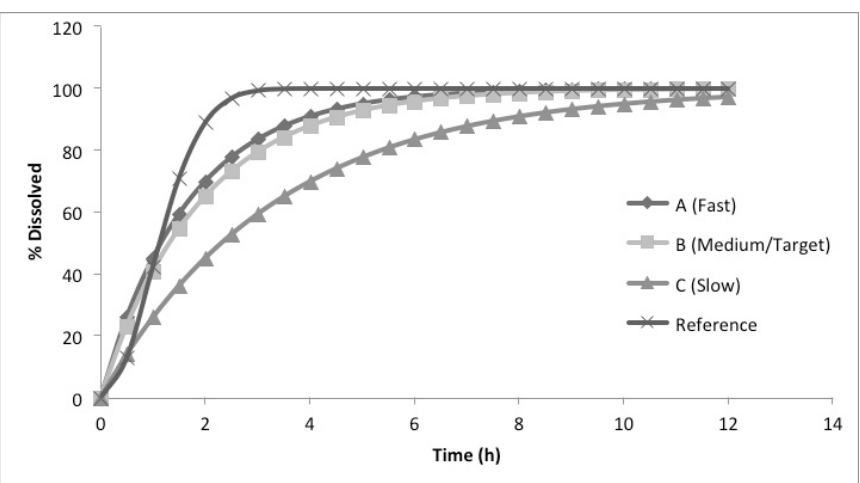

Figure 5. In vitro dissolution percentage dissolved of test formulations A (Fast), B (Medium/Target), and C (Slow) compared with the reference formulation using the new dissolution method.
This new dissolution test displayed the correct ranking among all formulations and highlighted the difference in behavior (mechanism of release) of the reference formulation versus all the test formulations. Results obtained with the new dissolution method were used to perform an IVIVC with the in vivo percentage absorbed already available including a common time scaling for all formulations (Figure 6).

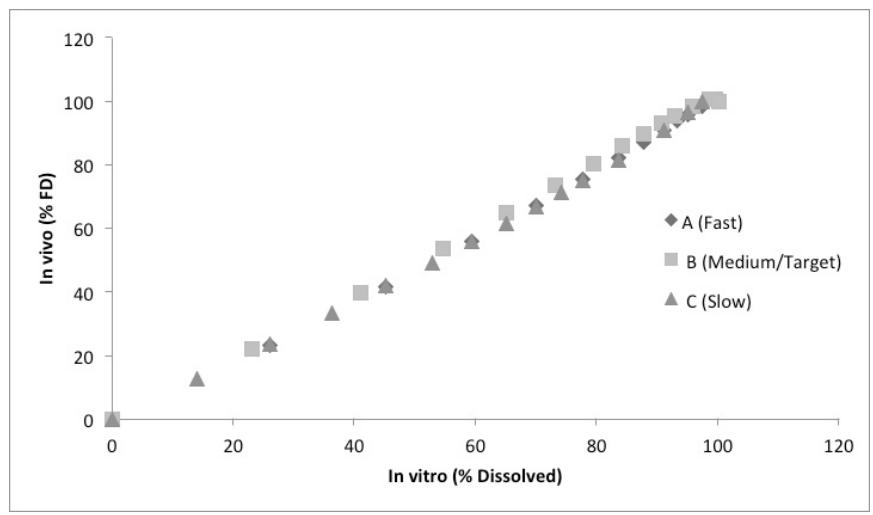

Figure 6. IVIVC between percentage dose absorbed from in vivo profiles vs. in vitro percentage dose dissolved with the new dissolution method for test formulations $\mathrm{A}$ (Fast), B (Medium/Target), and C (Slow).

The first outcome of this new IVIVC was the common relationship for all formulations suggesting that a similar limiting factor exists for all of them and might be related to the common release mechanism of the formulations. The equation of the curve is of the form

$$
\text { vivo = slope } x \text { vitro }+ \text { intercept }
$$

The predictability calculated denoted the validity of the established IVIVC.

\section{USING IVIVC TO ESTABLISH THE IDEAL PROFILE TO MATCH THE REFERENCE FORMULATION}

IVIVC (mainly Level A) is a powerful tool in drug development that can be used in many different instances (5-11). It shows a good understanding of the release characteristics of the pharmaceutical dosage form over time. The European guidelines $(5,6,11)$ and FDA guidelines (7-11) emphasize the interest in IVIVC and the use of IVIVC to optimize formulations, set dissolution limits, reduce the number of bioequivalence studies during product development, and facilitate certain regulatory decisions.

Based on the IVIVC expressed by the previous equation and on the calculated in vivo absorption profile of the reference formulation (Figure 3 ) the optimal dissolution curve, using the technology/formulation of the test formulation, could be derived using:

$$
\text { vitro }_{\text {ref }}=\left(\text { vivo }_{\text {ref }}-\text { intercept }\right) / \text { slope }
$$

This calculated optimal in vitro dissolution curve represents the dissolution that must be obtained by the new formulation using the technology of the test formulations. According to the type of technology 
(coating, matrix system, multiparticulate dosage form, specific excipient, etc.), the key factors of the formulation (critical quality attribute: CQA) will be used to develop the best formulation that will match exactly with the in vitro requirements. For example, for a coated technology, parameters such as amount of coating, type of plasticizer, type of coating polymer, and coating thickness are CQAs that could be modified and tested to reach the target in vitro profile. Based on the defined parameters, a DOE is established to optimize the formulation. Various types of DOE could be used from a factorial design to a simplex method. This approach optimizes the formulation as well as reduces the risks of no bioequivalence in further studies $(1-4,16,17)$. Thus, an optimal dissolution curve could be different from the in vitro dissolution determined with the reference using the defined in vitro conditions as: (1) the dissolution test was set up for the test formulation, (2) dissolution can be over- or under-discriminating, but this factor is accounted for by the IVIVC, and (3) the selected technology could also influence the way to obtain the optimal formulation, an exact copy of the reference.

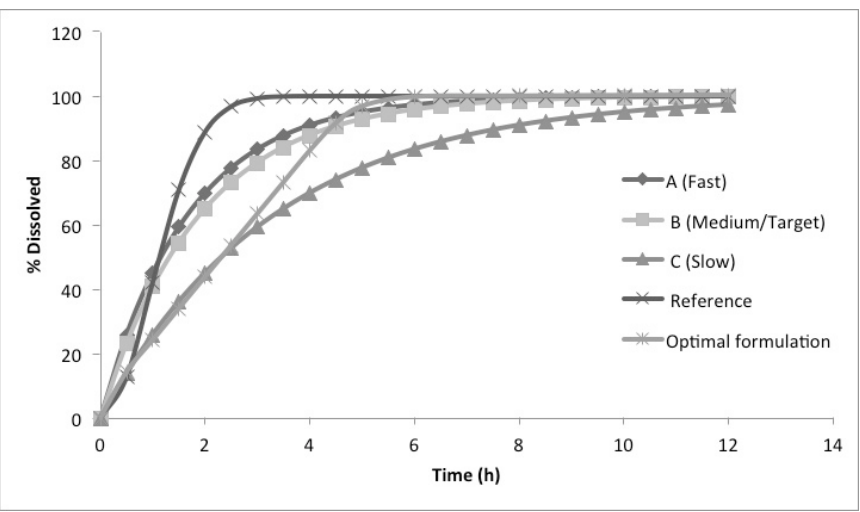

Figure 7. Optimal in vitro percentage dissolved compared with in vitro dissolution of test formulations A (Fast), B (Medium/Target), and C (Slow) and reference using the new dissolution method.

In the present example, the target dissolution, even if mimicking the reference profile, differs in rate. Results of the DOE allowed the development of a new formulation and calculation of the chance of in vivo success based on the IVIVC. IVIVC may reduce the number of in vivo studies during drug development and can be established using forecasted studies with no additional cost.

\section{CONCLUSIONS}

IVIVC links in vitro dissolution profiles and in vivo data and can be used classically to simulate the in vivo performance of a dosage form based on in vitro data. On the other hand, an optimal dissolution curve can be determined based on the in vivo data and the established IVIVC. This optimal dissolution curve can be the target curve on which to optimize formulations based on a DOE.

\section{REFERENCES}

1. Cardot, J.-M.; Beyssac, E. In vitro/in vivo correlations:
Scientific implications and standardisation. Eur. J. Drug Metab. Pharmacokinet. 1993, 18 (1), 113-120. DOI: 10.1007/BF03220014.

2. Cardot, J.-M.; Beyssac, E. In Vitro/In Vivo Correlation. In Encyclopedia of Pharmaceutical Technology, 3rd ed.; Swarbrick, J., Boylan, J. C., Eds.; Informa Healthcare: New York, 2006;. Vol. 1, pp 2062-2072. DOI: 10.3109/9780849393983.149.

3. Guideline on the Investigation of Bioequivalence; CPMP/ EWP/QWP/1401/98 Rev. 1; Committee for Medicinal Products for Human Use (CHMP), European Medicines Agency: London, 2010.

4. Cardot, J.-M.; Beyssac, E.; Alric, M. In Vitro-In Vivo Correlation: Importance of Dissolution in IVIVC. Dissolution Technol. 2007, 14 (1), 15-19. DOI: 10.14227/ DT140107P15.

5. Guideline on quality of oral modified release products; EMA/CHMP/QWP/428693/2013, Committee for Medicinal Products for Human Use (CHMP), European Medicines Agency: London, 2013.

6. Guideline on the pharmacokinetic and clinical evaluation of modified release dosage forms; EMA/CPMP/ EWP/280/96 Corr1, Committee for Medicinal Products for Human Use (CHMP), European Medicines Agency: London, 2014.

7. Waiver of In Vivo Bioavailability and Bioequivalence Studies for Immediate-Release Solid Oral Dosage Forms Based on a Biopharmaceutics Classification System; Guidance for Industry; U.S. Department of Health and Human Services, Food and Drug Administration, Center for Drug Evaluation and Research (CDER), U.S. Government Printing Office: Washington, DC, 2000.

8. Bioavailability and Bioequivalence Studies for Orally Administered Drug Products-General Considerations; Guidance for Industry; U.S. Department of Health and Human Services, Food and Drug Administration, Center for Drug Evaluation and Research (CDER), U.S. Government Printing Office: Washington, DC, 2003.

9. Extended Release Oral Dosage Forms: Development, Evaluation, and Application of In Vitro/In Vivo Correlations; Guidance for Industry; U.S. Department of Health and Human Services, Food and Drug Administration, Center for Drug Evaluation and Research (CDER), U.S. Government Printing Office: Washington, DC, 1997.

10. Immediate Release Solid Oral Dosage Forms, Scale-Up and Postapproval Changes: Chemistry, Manufacturing, and Controls, In Vitro Dissolution Testing, and In Vivo Bioequivalence Documentation; Guidance for Industry; U.S. Department of Health and Human Services, Food and Drug Administration, Center for Drug Evaluation and Research (CDER), U.S. Government Printing Office: Washington, DC, 1995.

11. InternationalConference on Harmonisation ofTechnical Requirements for Registration of Pharmaceuticals for Human Use. Pharmaceutical Development, Q8(R2); ICH 
Harmonised Tripartite Guideline: Geneva, Switzerland, 2009.

12. Pabst, $G$. Revalidation of the in vitro specification limits after an in vitro-in vivo correlation. Arzneim. Forsch. 1998, 48 (5A), 584-588.

13. Roudier, B.; Davit, B.; Schütz, H.; Cardot, J.-M. Impact of Data Base Structure in a Successful In Vitro-In Vivo Correlation for Pharmaceutical Products. AAPS J. 2015, 17 (1), 24-34. DOI: 10.1208/s12248-014-9680-x.

14. Siewert, $M$. Perspectives of in vitro dissolution tests in establishing in vivo/in vitro correlations. Eur. J. Drug Metab. Pharmacokinet. 1993, 18 (1), 7-18. DOI: 10.1007/BF03220004.

15. Martinez, M.N.;Amidon,G.L.AMechanistic Approach to Understanding the Factors Affecting Drug Absorption: A Review of Fundamentals. J. Clin. Pharmacol. 2002, 42 (6), 620-643. DOI: 10.1177/00970002042006005.

16. Hemmingsen, P. H.; Haahr, A.-M.; Gunnergaard, C.; Cardot, J.-M. Development of a New Type of Prolonged Release Hydrocodone Formulation Based on Egalet ${ }^{\circledR}$ ADPREM Technology Using In Vivo-In Vitro Correlation. Pharmaceutics 2011, 3 (1), 73-87. DOI: 10.3390/ pharmaceutics3010073.

17. Leeson, L. J. In Vitro/In Vivo Correlations. Ther. Innovation Regul. Sci. 1995, 29 (3), 903-915. DOl: $10.1177 / 009286159502900312$.
18. Devane, J. Impact of IVIVR on Product Development. In In Vitro-In Vivo Correlations; Young, D. B., Devane, J. G., Butler, J., Eds.; Advances in Experimental Medicine and Biology, Vol. 423; Plenum Press: New York, 1997; pp 241-260. DOI: 10.1007/978-1-4684-6036-0_23.

19. Dunne, A.; Gaynor, C.; Davis, J. Deconvolution Based Approach for Level A In Vivo-In Vitro Correlation Modelling: Statistical Considerations. Clin. Res. Regul. Aff. 2005, 22 (1), 1-14. DOI: 10.1081/CRP-54957.

20. Dunne, A.; O'Hara, T.; Devane, J. Approaches to IVIVR Modelling and Statistical Analysis. In In Vitro-In Vivo Correlations; Young, D. B., Devane, J. G., Butler, J., Eds.; Advances in Experimental Medicine and Biology, Vol. 423; Plenum Press: New York, 1997; pp 67-86. DOI: 10.1007/978-1-4684-6036-0_6.

21. Gillespie, W. R. Convolution-Based Approaches for in Vivo-in Vitro Correlation Modeling. In In Vitro-In Vivo Correlations; Young, D. B., Devane, J. G., Butler, J., Eds.; Advances in Experimental Medicine and Biology, Vol. 423; Plenum Press: New York, 1997; pp 53-65.

22. Cardot, J.-M.; Davit, B. M. In vitro-In Vivo Correlations: Tricks and Traps. AAPS J. 2012, 14 (3), 491-499. DOI: 10.1208/s12248-012-9359-0. 